\title{
16 Das Krim-Chanat. Osmanische Suzeränität und osteuropäisches Gleichgewicht
}

\begin{abstract}
Ich will Sie nun auch von der Macht und Regierungsform des Chans [...] ein wenig unterhalten. Laut Verträgen, die die Chane vor Zeiten mit der Pforte eingegangen sind, nimmt sich diese das Vorrecht, jene zu ernennen, und lässt dem tartarischen [sic!] Adel die Ehre ihn zu bestätigen, die alsdenn aus Furcht zu misfallen, selten etwas wider ihren neuen Chan einwenden. Wenn aber der türkische Hof von der tartarischen Nation bey einem Krieg [...] auBerordentliche Hilfe haben will, so giebt derselbe zuweilen aus politischen Ursachen dem Adel die Ehre, ihren Fürsten zu erwählen, und bestätigt ihn. ${ }^{1}$
\end{abstract}

Die obigen Zeilen stammen aus der Feder des habsburgischen Untertanen Nikolaus Ernst Kleemann (1736 - vermutlich nach 1800). ${ }^{2}$ Der Kaufmann reiste, wie dem Titel seines erstmals 1771 in Wien veröffentlichten Reiseberichts zu entnehmen ist, zwischen 1768 und 1770 in Richtung Schwarzes Meer und besuchte auch die Krim. Sogar eine Audienz bei einem der letzten Krim-Chane war ihm vergönnt, dem zwischen 1758 bis 1764 und 1768 bis 1769 herrschenden Kirım Giray. Und offenbar machte dieser auf den Handlungsreisenden Eindruck, schrieb Kleemann doch: „Niemals soll dieses Land einen größeren, klügeren, und von seiner Nation geliebtern Beherrscher gehabt haben, als den Crimm Gerey Chan, mit dem ich selbst gesprochen habe.“ Er lobte dessen „Verstand, seine Geschicklichkeit und Erfahrung im Krieg."3

Bereits wiederholt wurde das komplexe Verhältnis zwischen Istanbul und Bağçasaray angesprochen, das der ungarische Frühneuzeithistoriker Sándor Papp berechtigt als eines „der großen Themen der Erforschung des Krimkhanats“ bezeichnet hat. ${ }^{4}$ Im Folgenden wird die konkrete Ausgestaltung der osmanischkrimtatarischen Verbindung genauer in den Blick genommen. Nach Kleemann herrschte im Chanat zwischen dem Sultan, der das Recht zur Ernennung des Chans hatte, dem Chan selbst und den Mirza, also dem tatarischen Adel, eine Art Dreiecksverhältnis. Letzteren gewähre der Herrscher der Krim, so heißt es, die Ehre, ihn zu bestätigen. Der Adel wisse dies offenbar zu schätzen, so zumindest die Einschätzung des habsburgischen Untertanen, und bemühe sich seinerseits um gute Beziehungen mit Bağçasaray. Eine zentrale Komponente im Verhältnis zwischen Istanbul und dem Chanat war bekanntlich die Beistandspflicht des

1 Kleemann (1771), 155.

$2 \mathrm{Zu}$ den wenigen vorhandenen biographischen Angaben vgl. Nikolaus Kleemann (2018).

3 Kleemann (1771), 150.

4 Papp (2012).

○ OpenAccess. (C) 2020 Kerstin S. Jobst, publiziert von De Gruyter. (cc) BY Dieses Werk ist lizenziert unter der Creative Commons Attribution 4.0 International. https://doi.org/10.1515/9783110520620-018 
letzteren im Kriegsfall bzw. bei Eroberungsfeldzügen, die Kleemann ebenfalls erwähnt. ${ }^{5}$ Zuweilen gab es offenbar Konstellationen - „wenn aber der türkische Hof von der tartarischen Nation bey einem Krieg [...] außerordentliche Hilfe haben will“ -, in denen der Sultan offenbar auf sein Recht, den Chan zu erwählen verzichtete, um sich im Gegenzug der Unterstützung der Mirza zu versichern. Er übertrug in solchen Fällen häufiger die Auswahl eines neuen Chans dem Adel. Anders als Broniewski ungefähr 150 Jahre vor ihm war Kleemann aber offenbar nicht bekannt, dass jeder Chan zwingend ein Giray sein und von Činggis Qayan abstammen musste. Das nicht immer spannungsfreie Verhältnis zwischen der Hohen Pforte, den Chanen und den Mirza war ihm hingegen nicht entgangen.

In multiethnischen Großreichen wie dem Osmanischen Reich gab es regelhaft eine Vielzahl differierender Rechtsbeziehungen zwischen der Metropole ${ }^{6}$, d.h. im konkreten Fall Istanbul, und den sog. Peripherien. ${ }^{7}$ In Bezug auf das Krim-Chanat ist festzustellen, dass es „im Vergleich mit anderen Reichsteilen eine bevorzugte Position“ innehatte. ${ }^{8}$ Während sich auf der bereits oben geschilderten symbolischen Ebene daran über die Zeit nichts Wesentliches änderte, wandelte es sich jedoch auf derjenigen der realen Politik: Mehr als ein Jahrhundert nachdem sich das Chanat im Jahr 1478 in Abhängigkeit zur Hohen Pforte begeben hatte, war es die Regel gewesen, dass der allerdings vetoberechtigte Sultan sein Einverständnis zur Einsetzung eines neuen, ihm von den Mirza vorgeschlagenen Chans gab. Eine Zäsur, so Papp, der den Inaugurationsprozess der Krim-Chane untersucht hat, vollzog sich allerdings zwischen 1608 und $1628 .{ }^{9}$ In dieser Zeit vergrößerte sich der Einfluss der osmanischen Herrscher, die nunmehr den präsumtiven Chan direkt ein- bzw. absetzen konnten. Ein jeder Kandidat musste sich nun seine sog. Bestallungsurkunde bei der Hohen Pforte persönlich abholen, musste also nach Istanbul reisen und dem Sultan gegenübertreten. Diese Prozedur sollte einerseits ein besonderes Loyalitäts- und Treueverhältnis begründen, ${ }^{10}$ kann aber andererseits auch als Unterwerfungsgeste gedeutet werden, musste der rangniedere Partner doch beim ranghöheren (also dem Sultan) um seine Inauguration ansuchen. In jedem Fall vergrößerte sich im Verlauf des 17. Jahrhunderts der Einfluss

5 Vgl. dazu beispielsweise Andreev (1997), 123.

6 Vgl. dazu Barkey (2008).

7 Das Begriffspaar Metropole - Peripherie wird hier nicht im Sinne einer kritiklosen Übernahme der sog. Dependenz-, aber auch der Weltsystemtheorie o.ä. verwendet, sondern einfach um räumliche und kulturelle Disparitäten zu benennen, ohne diese werten zu wollen. $\mathrm{Zu}$ den Begrifflichkeiten vgl. einführend Boeckh (1993); Nölke (2006).

8 Jobst (2011b), 16.

9 Papp (2012), 77, dem ich auch im Weiteren überwiegend folge.

10 Vgl. Papp (2012), $83 \mathrm{f}$. 
Istanbuls, das nun auch die inneren Verhältnisse im Chanat wesentlich mitbestimmen konnte. ${ }^{11}$ Dies hatte der aus dem Osmanischen Reich stammende Evliyâ Çelebi (s.o.) während seines Krim-Aufenthaltes ja durchaus bemerkt. ${ }^{12}$

Bereits die ersten Krim-Chane, Hacı I., Nur Devlet und Mengli I. Giray, konnten nicht ohne Unterbrechung herrschen, was allerdings nicht auf das Zutun der Hohen Pforte zurückzuführen war, sondern auf Konflikte innerhalb der Krim. Ab dem 17. Jahrhundert nutzten die Sultane aber regelmäßig ihr Recht auf Einoder Absetzung eines Chans, um ,den eigenen, nicht immer gehorsamen Vasallen in Schach zu halten. "13 Zuweilen nutzte allerdings zur Durchsetzung Istanbuler Interessen nicht allein das Wort des Sultans, so dass auch militärische Mittel gegen die Krim-Herrscher eingesetzt werden mussten. ${ }^{14}$ Istanbul machte zudem, und darauf hat Kleemann in seiner Beschreibung der Machtkonstellation auf der Halbinsel hingewiesen, von der Macht der Mirza Gebrauch. Insbesondere Clans wie die Barın oder Şirin waren in der Lage, beim ,Loswerden` eines eigensinnigen oder aus anderen Gründen missliebigen Herrschers in Bağçasaray zu helfen. Manchmal bot die Hohe Pforte Chanen sogar finanzielle Belohnungen, damit diese ihre Absetzung akzeptierten. ${ }^{15}$ Matuz berichtet, dass Geiselpolitik eine weitere Maßnahme war, um die politischen Entwicklungen auf der Krim im Sinne des Sultans zu gestalten: Dazu wurden Angehörige der Girays im Topkapı-Palast in Istanbul festgehalten, um gegebenenfalls den osmanischen Forderungen mehr Nachdruck verleihen zu können, indem man drohte, die ,Gäste‘ zu töten oder schlecht zu behandeln. ${ }^{16}$ Ein Nebeneffekt dieser Praxis war, präsumtiven KrimChanen während ihres erzwungenen Aufenthaltes in Istanbul eine Erziehung im Sinne der Osmanen angedeihen zu lassen. ${ }^{17}$

Die besondere Wertschätzung, die dem Chanat seitens der Osmanen - und übrigens auch später durch die zarische Macht (vgl. hierzu Kapitel 22 und 23) entgegengebracht wurde, basierte nicht unwesentlich auf dessen militärischer Stärke. Die daraus resultierende Verpflichtung zum Waffendienst der Krimtataren an der Seite der Osmanen hatte eine ganze Reihe von Nebeneffekten. Einer davon - und noch im Detail zu betrachten - war das zwischen Angst und Bewunderung changierende Krimtatarenbild im kollektiven europäischen Gedächtnis. Ein weiterer Effekt war finanzieller Art, hatten die Chane und ihre Krieger doch konkrete

\footnotetext{
11 Papp (2012), 76.

12 Vgl. Hillebrand (2017), u. a. 54.

13 Matuz (1964), 134.

14 Vgl. Papp (2012), 79.

15 Papp (2012), $79 \mathrm{f}$.

16 Vgl. Matuz (1964), 135.

17 Matuz (1964),135.
} 
ökonomische Vorteile: Nicht nur, dass gegenüber dem Osmanischen Reich keine Tributpflicht bestand - im Gegenteil, die Herrscher in Bağçasaray erhielten nicht allein aufgrund ihrer allseits geschätzten Abkunft von Činggis Qayan offenbar recht großzügige Zahlungen und Belohnungen von Istanbul, sondern auch wegen der allseits anerkannten Geschicklichkeit krimtatarischer Soldaten auf dem Felde. Kein Sultan wollte oder konnte auf solch gute Kämpfer verzichten. Die Übertragung umfänglichen Landbesitzes an die Krim-Chane in anderen Teilen des Osmanischen Reiches, eine Art jährliche Pension oder auch eine vom Sultan bezahlte Leibgarde (sekban genannt) waren weitere Belohnungen, durch die das Band zwischen der Halbinsel und Istanbul gefestigt wurde. ${ }^{18}$ Die Aufstellung der sog. sekban zum Schutz des Chans erwies sich als eine besonders durchdachte Maßnahme, hatte Istanbul doch damit im Zweifels- bzw. Konfliktfall vom Sultan finanziell abhängige (und damit loyale) bewaffnete Kräfte in unmittelbarer Nähe des Chans positioniert. In jedem Fall gewährte Istanbul den Krim-Chanen im Vergleich mit anderen Teilen des Imperiums großzügige Zuwendungen. ${ }^{19}$ Auch von der Hohen Pforte wurde das Chanat als eigentlicher Erbe der Horde angesehen, denn der Chan durfte weiterhin die jährlichen Tribute (pl. upomniki; russ. pominki) kassieren, die das Moskauer Reich und Polen-Litauen seit den Zeiten der Goldenen Horde entrichten mussten.

Durch den Einsatz tatarischer Hilfstruppen im Verbund mit der Hohen Pforte erweiterte sich der militärische Aktionsradius des Krim-Chanats enorm. ${ }^{20}$ Bei Militäraktionen des Osmanischen Reiches gegen Ungarn oder Persien beispielsweise waren sie genauso involviert wie bei der sog. Ersten (1529) und Zweiten Wiener Türkenbelagerung (1683). ${ }^{21}$ In einer wissenschaftlich akzeptablen, aber sprachlich von stereotypen Vorstellungen über eine ,den Orientalen` eigene Grausamkeit nicht freien Abhandlung aus dem Jahre 1976 ist beispielsweise zu lesen, dass die krimtatarischen Reiter vor Wien ganz in der Art ihrer aus den eurasischen Steppen stammenden Vorväter aufgetreten seien: Sie hätten eine Spur der Zerstörung hinterlassen, die Europa seit der Invasion Batu Chans in Zentraleuropa in der Mitte des 13. Jahrhunderts nicht mehr erlebt habe. ${ }^{22}$ Un-

18 Faroqhi (1994), 419, definiert diese als „mercenaries of peasant background.“

19 Dazu genauer: Fisher (1972a).

20 Hierzu exemplarisch: Petritsch (1983).

21 Vgl. Kołodziejczyk (2012), 50. Insbesondere zur Wahrnehmung krimtatarischer Soldaten 1683 durch die autochthone Bevölkerung vgl. Augustynowicz (2012).

22 Barker (1967), 220. Die Mehrheit der HistorikerInnen sieht die Teilnahme krimtatarischer Truppen an der Zweiten „Türkenbelagerung“ von Wien als erwiesen an. Der als absoluter Kenner krimtatarischer Außenpolitik in der Frühen Neuzeit geltende Kołodziejczyk (2012), 51, geht hingegen davon aus, dass der damalige Krim-Chan Murad Giray (1627-1696) seine Teilnahme an 
strittig ist, dass es im Fall der Belagerung Wiens 1683 auch zu von Krimtataren begangenen Gewalttätigkeiten - ebenfalls gegen Zivilpersonen - gekommen war. ${ }^{23}$ Doch ist Gewalt militärischen Konflikten inhärent und kann nicht als quasiprimordiales Kennzeichen bestimmter Kulturen oder Großgruppen angesehen werden. Insgesamt gilt, dass die „Beteiligung des Krim-Khanates am Türkenkrieg von 1683-1699 [...] noch immer nicht ausreichend untersucht worden“ ist. ${ }^{24}$

Grundsätzlich erwartete die Hohe Pforte, dass das Chanat seine Außenpolitik an die Istanbuls anpasste bzw. zumindest mit dieser abstimmte. ${ }^{25}$ Häufig entzogen die Chane sich diesem Anspruch jedoch und entwickelten eigenständige Interessen, die sie umzusetzen suchten. Mehr oder weniger unabhängige ,außenpolitische' Verbindungen unterhielt das Chanat z. B. innerhalb des Osmanischen Reiches, etwa zu den Donaufürstentümern Moldau und Walachei, die ihrerseits zwar gleichsam Vasallen des Sultans waren, allerdings ebenfalls über einen bevorzugten Autonomiestatus verfügten. ${ }^{26}$ Aber auch außerhalb des Reichszusammenhangs trat das Krim-Chanat immer wieder als eigenständiger Akteur in Erscheinung: So unterhielt es phasenweise eigene diplomatische Beziehungen zu den Königreichen Dänemark und Schweden. ${ }^{27}$ Regelhaft gab es diplomatische Kontakte und krimtatarische Gesandtschaften an die großen europäischen Höfe wie Wien $^{28}$ und Moskau ${ }^{29}$ oder seit dem ausgehenden 16. Jahrhundert auch zu im Aufstieg begriffenen Mächten wie Brandenburg-Preußen. ${ }^{30}$ Außer bei den bereits dargestellten Eingriffen der Sultane bei der Einsetzung der Chane und der daraus resultierenden Beeinflussung der Innenpolitik des

diesem Feldzug verweigert hat. Unbestritten ist, dass er dieser Aktion ablehnend gegenüberstand, da er sie für wenig erfolgversprechend hielt; vgl. Cardini (2004), 234. Zu den Hintergründen der Entscheidung des Großwesirs Kara Mustafa vgl. immer noch Leitsch (1981).

23 Vgl. dazu im Überblick auf der Grundlage von Augenzeugenberichten Augustynowicz (2012). 24 Schwarcz (2017), 208. Schwarcz erklärt dies mit der komplexen Quellenlage, ist das entsprechende Material doch in Istanbul, Wien, Moskau, Warschau, Paris und Teheran und zudem nur teilweise ediert.

25 Vgl. Andreev (1997), 123.

26 Vgl. hierzu Arens u. Klein (2004), 497. Das Fürstentum Siebenbürgen konnte ebenfalls den Status eines privilegierten Vasallen der Hohen Pforte beanspruchen, ,und zwar zum einen durch den ihnen seitens Istanbul zugestandenen inneren und äußeren politischen Aktionsradius, zum anderen hinsichtlich ihrer Tributleistungen." Moldau und die Walachei waren durch wesentlich höhere Tribute als das Krim-Chanat oder Siebenbürgen belastet.

27 Vgl. hierzu die überaus verdienstvolle Edition und Untersuchung Matuz (1976).

28 Vgl. dazu Augustynowicz (2005).

29 Diese wechselseitigen Kontakte zwischen Moskau und Bağçasaray dienten nicht allein der Übergabe der Tribute, sondern können wohl auch als ,normale‘ diplomatische Kontakte mit üblichen Konflikten interpretiert werden. Vgl. auch Zercalov (1890).

30 Dazu Hottop-Riecke (2017), besonders $69 \mathrm{f}$. 
Chanats (s.u.) entstanden auf dem Feld der Außenpolitik Unstimmigkeiten - und zwar dann, wenn der krimtatarische Aktionsradius durch die Politik der Osmanen beschränkt werden sollte; dies war vor allen Dingen dann der Fall, wenn die Osmanen sich in den Steppengebieten des nördlichen Schwarzmeerraums ausbreiteten, die wiederum von den Krimtataren als ihre ureigene Interessenszone betrachtet wurden, handelte es sich dabei doch um Territorien, die ehemals mittelbar oder unmittelbar zur Horde gehört hatten. Wenn das Osmanische Reich Maßnahmen gegenüber den nördlichen Nachbarn des Chanats, also Polen-Litauen und dem Moskauer Staat, ergriff, kam es besonders häufig zu Konflikten. Nachdem beispielsweise ein Großteil des zu Polen-Litauen gehörenden Podoliens während des Osmanisch-Polnischen Krieges von 1672 bis 1676 an die Osmanen gefallen war, verschlechterte sich das Verhältnis zum Vasallen auf der Halbinsel rapide: Das Chanat hatte dadurch nämlich keinen Zugriff mehr auf eine ökonomisch wichtige Region, hatte es sich doch in Podolien über lange Zeit ein stark nachgefragtes Handelsgut besorgt: Sklavinnen und Sklaven. ${ }^{31}$ Festzuhalten ist, dass es im 17. und frühen 18. Jahrhundert vermehrt Absetzbewegungen des Chanats von der Hohen Pforte gab, deren Ziel die Herauslösung aus dem osmanischen Reichsverband war. Sie blieben aber erfolglos. ${ }^{32}$

Der russische Orientalist Smirnov hatte schon im 19. Jahrhundert erkannt, nach welcher ,Regel‘ die Herrscher in Bağçasaray auf dem Gebiet der Außenpolitik gegenüber Polen-Litauen und Moskau vorgingen: Sie unterstützten vorzugsweise den jeweils Schwächeren der beiden, ${ }^{33}$ was in heutiger Diktion wohl als Politik des Gleichgewichts bezeichnet werden würde. Wenn also dieses Gleichgewicht aus der Perspektive Bağçasarays gestört zu werden drohte, resultierten daraus zuweilen Fälle von Ungehorsam gegenüber der Hohen Pforte. Das Chanat unter Devlet Giray (1512-1577) nahm 1569 gemeinsam mit den Osmanen an dem wie sich zeigen sollte - erfolglosen Feldzug gegen das 1556 von Moskau eroberte ehemalige Chanat von Astrachan' teil. Da Devlet Giray sich wie seine Vorgänger im Palast von Bağçasaray als einziger legitimer Erbe der Mongolen sah, hatte er sich wie diese dem sog. Sammeln der Länder der Goldenen Horde verschrieben; sein Ziel war die Verdrängung des Moskauer Zarentums aus der Region, welches er hoffte an der Seite der Osmanen erreichen zu können. Als die osmanische Macht aber Anstalten machte, in der Steppe den Bau eines den Don und die Wolga verbindenden Kanals in Angriff zu nehmen, der den Einfluss des Sultans auf die von den Krimtataren beanspruchten Gebiete gestärkt hätte, änderte sich die

31 Zum konkreten Ereignis Kołodziejczyk (2012), 52.

32 Vgl. Fisher (1977).

33 Smirnov (1887), 555. 
Sachlage. Istanbul plante nämlich mittelfristig die Verlegung der osmanischen Schwarzmeer-Flotte ins Kaspische Meer und versprach sich durch den Kanalbau massive wirtschaftliche Vorteile. ${ }^{34}$ Als Devlet dessen während des Kriegszuges gegen Astrachan' gewahr wurde, verließ er mit seinen Truppen die Militärkampagne ,in the midst“ (also mittendrin), so Fisher, irgendwo an der Wolga. ${ }^{35}$ In der krimtatarischen Erinnerungskultur hätte er sich allein mit diesem Eigensinn einen Platz verdient, geradezu unsterblich wurde Devlet aber auch im russischen kollektiven Gedächtnis. Dort kommt ihm allerdings eine negative Rolle zu, verantwortete er doch den verheerenden Raubzug gegen Moskau, in dessen Folge die Stadt im Mai 1571 niedergebrannt wurde. Nikolaj M. Karamzin (1766-1826), der bedeutende russische Schriftsteller, Sprachreformer und offizielle Reichshistoriograph am Zarenhof, beschrieb in seiner einflussreichen „Geschichte des Russländischen Staates“ (1816-1829) dieses Ereignis folgendermaßen:

\begin{abstract}
Devlet-Girey vollbrachte eine große Tat [podvig]: Er wollte nicht den Kreml belagern, und von den Sperlingsbergen aus betrachtete er seinen Triumph, Haufen rauchender Asche im Umkreis von dreißig Werst, sofort entschied er zurückzugehen, aus Angst [...] vor dem unzutreffenden Gerücht, dass der Fürst oder Große König sich mit einer großen Armee nähert. Ioann [=Ivan IV., ,,der Schreckliche“] hatte in Rostov die Botschaft vom Rückzug des Feindes erhalten, und befahl Fürst Vorotynskij dem Chan zu folgen, dem es allerdings gelang, den Großteil der südöstlichen Regionen Moskaus zu zerstören und mehr als 100.000 Gefangene nach Taurien zu bringen. Nicht die Großherzigkeit habend, der Tröster seiner Untertanen im Moment der schrecklichen Katastrophe zu sein, [und] ängstlich, den Schauplatz des Schreckens und der Tränen sehen zu müssen, wollte der Zar nicht in die niedergebrannte Hauptstadt. [...] Es gab niemanden, der die Toten beerdigen konnte. Er [Ivan] befahl, Moskau von den verwesenden Leichen zu befreien. [...] Nur Adlige und Reiche wurden nach dem christlichen Ritus beerdigt. Die Körper der Anderen füllten die Moskva, so dass der Fluss nicht mehr fließen konnte. Sie lagen in Haufen, vergifteten das Wasser und die Luft durch die Fäulnis; und die Brunnen waren ausgetrocknet und ohne Wasser. Die übrig gebliebenen Bewohner vergingen vor Durst. Am Ende holten sie das Volk aus den umliegenden Städten; sie zogen die Leichen aus dem Fluss und begruben sie. So hat sich der endlose Zorn des Himmels über Russland ergossen. ${ }^{36}$
\end{abstract}

In diesen Zeilen wird, neben der sich durch Karamzins Werk ziehenden Kritik an Ivan IV. (1530-1584), ${ }^{37}$ der nicht die Größe gehabt habe, „der Tröster seiner Un-

34 Matuz (1996), 139.

35 Fisher (1978), 45.

36 Karamzin (2013).

37 Der das autokratische System grundsätzlich rechtfertigende Karamzin mag sich bei seinen deutlichen Vorbehalten gegenüber Ivan IV. von der Gewalt der Opričniki, einer speziellen Militäreinheit des Zaren, leiten lassen haben. Hosking (1997), 87, ist der Auffassung, dass die durch 
tertanen im Moment der schrecklichen Katastrophe zu sein“, ${ }^{38}$ eine Zerstörung fast apokalyptischen Ausmaßes geschildert: eine durch die Krimtataren weithin verheerte Stadt, von der allein der Kreml verschont geblieben war, überall Leichen, die niemand beerdigen konnte. Und die Davongekommenen mussten den Weg in die Sklaverei nach Taurien, also auf die Krim, antreten - all das interpretierte Karamzin als göttliche Strafe, welche die russischen Länder heimgesucht hätte.

Der Einfall der Krimtataren in das Moskauer Reich von 1571 war weder der erste noch der letzte, aber wohl der verheerendste seiner Art. Gerade im 16. Jahrhundert und im Kontext des Kampfes um das Erbe der Goldenen Horde kam es immer wieder zu überraschenden Vorstößen krimtatarischer Truppen nach Norden; genau fünfzig Jahre vorher, im Sommer 1521, hatte es bereits eine unerwartete Kampagne gegeben, bei dem Truppen von der Krim fast bis nach Moskau gelangt waren, ehe sie sich wieder zurückzogen. Dies stand übrigens im Zusammenhang mit den krimtatarischen Versuchen, eine dauerhafte Präsenz des Moskauer Reiches an der Wolga zu verhindern. Seit 1521 war es dem Krim-Chanat wiederholt gelungen, den Chan-Thron in Kazan' mit Girays zu besetzen. Damit hatte die seit 1487 währende erste Einnahme dieses Chanats durch Ivan III. (1440-1505) vorerst beendet werden können, was den Krimtataren in Allianz mit dem Chanat von Astrachan' und den Nogaiern gelungen war. ${ }^{39}$ Doch währte die Freude darüber nicht lange, nahm Ivan IV. doch 1552 Kazan’ ein - und konnte es diesmal dauerhaft für sein Reich sichern.

In jedem Fall zeigen die genannten Beispiele, dass das Krim-Chanat durchaus als eigenständiger Akteur gegenüber den Osmanen auftreten konnte - und auftrat! Wie sind nun abschließend der Spielraum und die Position des Krim-Chanats in der Frühen Neuzeit in der nördlichen Schwarzmeerregion und sein Einfluss bis ins östliche und ostmitteleuropäische Europa hinein zu bewerten? In der Forschung gibt es divergierende Meinungen, so sieht der russische Historiker Ilya Zaytsev die Macht der Chane immer auch als „a reflection of the power of the Ottoman padişah [Sultan].“40 Dies ist insofern richtig, als dass ein mächtiger Sultan - wie gezeigt - sich leichter tat, einzelnen Thronprätendenten aus der Familie der Girays die Macht zu geben und wieder zu entziehen, als ein schwacher Sultan. Aufgrund ihrer reklamierten Abkunft von Činggis Qayan und des gemeinsamen Glaubens besaßen die Chane in den Augen des Hofes von Istanbul

Gesetzlosigkeit korrumpierte und geschwächte Truppe Ivans IV. 1571 unfähig gewesen sei, „den Angriff der Truppen des Dewlet-Girei abzuwehren.“

38 Vgl. grundlegend Hoffmann P. (1991).

39 Fisher (1978), $40 \mathrm{f}$.

40 Zaytsev (2010), 25. 
aber in jedem Fall auch einen Ehrenvorteil gegenüber den meisten anderen Vasallen. ${ }^{41}$ Das zeigt sich u.a. daran, dass nicht wenige Girays dort hohe Positionen bekleideten. Dass die übrigen KrimtatarInnen aus der Perspektive der Eliten des Osmanischen Reiches als primitiv und rückständig angesehen wurden, als islamisierte Barbaren, „whose task was to protect the northern border of the Empire“, so Zaytsev ${ }^{42}$, spricht zumindest für deren Wertschätzung auf dem Gebiet des Kampfes, wenn auch nicht auf dem der nichtmilitärischen Kultur. Welche Vorstellungen über krimtatarische Unterschichten osmanische Eliten pflegten, wäre in jedem Fall weitere Untersuchungen wert.

Das Verhältnis zwischen dem Krim-Chanat und dem Osmanischen Reich war über einen langen Zeitraum eher das eines zwischen Verbündeten denn zwischen Vasall und Lehnsherr, bewiesen seine Eliten doch vielfach Eigensinn und den Willen, eine von der Pforte unabhängige Politik nach innen (s.u.) und außen durchzusetzen. Auch wenn sich der Gestaltungsspielraum über die Zeit verringert haben mag, so ist dem Urteil Fishers über das Chanat zuzustimmen: „The Crimean Tatar Khanate was one of the most important states in eastern Europe from the early sixteenth century until the end of the seventeenth century. “43

41 Dies belegte u.a. der von Hillebrand (2017) untersuchte Reisebericht des osmanischen Untertanen Evliyâ Çelebi.

42 Zaytsev (2010), 25.

43 Fisher (1978), 17. 
